\title{
An MDA Method for Automatic Transformation of Models from CIM to PIM
}

\author{
Abdelouahed Kriouile, Najiba Addamssiri, Taoufiq Gadi
}

LAVETE Laboratory, Hassan 1 University, Settat, Morocco

Email address:

kriouile1970@gmail.com (A. Kriouile), addam.naji@gmail.com (N. Addamssiri),g.taoufiq@yahoo.fr (T. Gadi)

To cite this article:

Abdelouahed Kriouile, Najiba Addamssiri, Taoufiq Gadi. An MDA Method for Automatic Transformation of Models from CIM to PIM. American Journal of Software Engineering and Applications. Vol. 4, No. 1, 2015, pp. 1-14. doi: 10.11648/j.ajsea.20150401.11

\begin{abstract}
The Model Driven Architecture (MDA) approach introduces a clear separation of the business logic from the implementation logic that's less stable. It uses the models that are more perennial than codes. It puts the models at the centre of the development of software and of the information systems. The MDA approach consists at, firstly, developing the CIM Model, secondly, obtaining the PIM model from the CIM, and finally generating the PSM model from the PIM which facilitates the generation of code for a chosen technical platform. In the literature, several works have summarized the MDA approach to the passage from PIM to PSM then from the PSM to code. Yet, very little work has contributed in the axis of the CIM to PIM transformation, and their approaches generally propose a CIM model which does not cover the different specifications of the Object Management Group (OMG) and/or the CIM to PIM transformation that they define is in the most cases manual or semi-automatic. Thus, our proposal aims at providing a solution to the problem of constructing CIM and its automatic transformation at the PIM using the QVT transformation rules. The approach proposes to represent CIM by two models: The business process model reflecting both the static and the behavioral views of the system, and the functional requirement model defined by the use case model reflecting the functional view of the system. The transformation of the CIM allows us to generate the PIM level represented by two models: The domain classes model which gives a structural view of the system at this level, and a model that describes the behavior of the system to each use case.
\end{abstract}

Keywords: MDA, CIM, PIM, Model Transformation, BPMN, QVT

\section{Introduction}

The discipline of software engineering has allowed the development of computer systems for more and more complex and requiring enormous investments. Yet, the sustainability of these systems is questioned whenever a new technology appears, since each new technology put in place a set of tools that, generally, does not support the older technologies.

However, in response to this difficult situation, we should reconsider the reduction of the cost of development work that is done independently from the target technology and which is, therefore, connected to the business logic of the application. The MDA approach (Model Driven Architecture) [1] is called by introducing a clear separation of the business logic -which is stable and undergoes little changes over timefrom the implementation logic that's less stable; models are more perennial than codes. This MDA approach fits into the overall context of the Model Driven Engineering (MDE), which puts the models at the centre of the development of software and of the information systems.

The principle of the MDA approach is based on the use of models and metamodels for the various phases of the software development lifecycle. Specifically, it recommends three types of models from different viewpoints) [1]: The Computation Independent Model (CIM), the Platform Independent Model (PIM) and the Platform Specific Model (PSM).

The MDA approach consists, at first, to develop the CIM Model. Secondly, to obtain the PIM model from the CIM, and finally to generate the PSM model from the PIM which facilitates the generation of code for a chosen technical platform. The passage from CIM to PIM and from PIM to PSM represents models transformations, while the generation of code from PSM is not regarded as a model transformation. Ideally, according to [1], the code generation can be done automatically by successive transformations of models: CIM to PIM, PIM to PSM and PSM to code.

In the literature, several works have summarized the MDA approach to the passage from PIM to PSM then from the 
PSM to code. Yet, very little work have contributed in the axis of the CIM to PIM transformation, and their approaches, generally, propose a CIM model which does not cover the different specifications of the Object Management Group (OMG) and/or the CIM to PIM transformation that they define is in the most cases manual or semi-automatic.

Thus, our proposal aims to be a new solution to the problem of constructing CIM and its automatic transformation at the PIM. This problem can be divided into two sub-problems:

- Elaborate a CIM model conforms to the various specifications outlined by the OMG.

- Define an automatic model transformation allowing generating the PIM model conform to the various specifications outlined by the OMG.

Thus, our initiative aims at solving or at least mitigating in the context of the MDA approach- the problems related to the sustainability of the applications. This sustainability cannot be ensured only through models that are sustainable and productive, independent from computing and independent from any technological platform. Our contribution seeks to solve this problem at top level of the MDA; it will specifically focus on modeling the CIM and define the transformation rules allowing generating the suitable PIM Model from the CIM Model. The transformations rules are expressed through language QVT.

In our approach, we propose to represent CIM by two models: The business process model (BPM) reflecting both the static aspect (Static View) and the behavioral aspect (Behavioral View) of the system, and the functional requirement model defined by the Use Case model (UC) reflecting the functional aspect (Functional View) of the system.

The transformation of the CIM allows us to generate the PIM model represented by two models: The domain classes model corresponds to the context in which the system should be applied which gives a static view of system (Structural View), and the Behavioral Model that describes the behavior of the system to each use case providing a behavioral view of system (Behavioral View).

The rest of this paper is organized as follows. In section 2, background and related work for this research are explained. Section 3 and section 4 depict respectively how the CIM and PIM will model. Section 5 presents the different steps of our method to construct CIM and to transform it automatically into PIM using the QVT transformation rules. In section 6, we shall present an illustrative case study. The analysis of the evaluation results of our proposal is explained in section7. Finally, in section 8 , we briefly provide a conclusion and present our plan for future works.

\section{Background and Related Work}

\subsection{Levels of Modeling in MDA}

The OMG announced its initiative MDA (Model Driven Architecture) in November 2000, then proposed its first version in 2001 and in 2003 adopted the final specification of the approach [2]. The MDA approach fits into the overall context of the model driven engineering (MDE), which puts the models at the centre of the development of software and of the information systems.

Since the models are more perennial than codes, and in order to enable the organizations to evolve their application models independently of the evolution of the technology platforms, MDA advocates the elaboration of perennial models by distinguishing models which are independent from the platforms of the models that are specific to platforms. Thus, its principle is to separate the functional specifications from the implementation specifications on a particular platform.

The MDA approach offers three types of models from three different points of view [1]:

- CIM (Computation Independent Model): It's a model independent of any computerization. The CIM Model does not show the details of the system structure. It describes the product independently of any computer system. It focuses on the requirements of the system as well as the environment in which it operates without going into the details of its structure and its implementation. It is sometimes called a domain model and serves as the vocabulary for system domain practitioners. However, a CIM is more than a domain model; it expresses also the requirements of the system.

- PIM (Platform Independent Model): It refers to a view of system or sub-system at an abstraction level allowing an independence from any technical platform. The role of the PIM is to be perennial and to make the link between the CIM and the PSM model. The MDA approach advocates the use of UML as a language to model the PIM. But it gives neither any indication of the number of models to develop at the PIM level nor the method to be used for developing it.

- PSM (Platform Specific Model): It is the dependent model of the technical platforms. It mainly serves as a base for generating an executable code on the chosen technical platform. It indicates how the product will be used on these platforms. A good PSM must incorporate enough features and concepts (data types, classes, interfaces, patterns, etc.) of the platform chosen to make the code generation easy.



Figure 1. MDA software lifecycle 


\subsection{Model Transformations in $M D A$}

The main artifacts of the MDA are models and model transformations. Generally, we call transformation of models any program which its inputs and outputs are models respectively conform to their metamodels. A model transformation matches, according to the transformation rules, the concepts of the source and the target metamodel. The transformation rules are described at the transformation models conform in turn to the metamodel that defines the transformation language. Thus, the elements of the target models can be generated from those of the sources models by applying transformation rules that are already defined.

In the MDA architecture the MOF (Meta Object Facility) allows the definition of modeling languages, as well as the definition of the transformation rules. It is normalized by the OMG in its current version 2.0 [3]. It is used to specify the structure and syntax of metamodels. It also specifies mechanisms for metamodel interoperability, allowing its comparing and its linking. Thanks to these exchange mechanisms, the MOF cohabit different metamodels.

The OMG proposes the standard MOF 2.0 QVT (Query View Transformation) [3] as language to define the transformation of models.



Figure 2. MDA processing Process

\subsection{Business Process Modelling}

BPMN and UML activity diagram are two competing standards, both maintained by the OMG, allowing to model business processes.

BPMN is based on a single Business Processes Diagram, called BPD [4]. It is easy to use and to understand, allowing modeling the complex business processes.

The UML AD specification does not deal with the business process modeling. It mentioned that the activities can be applied to organizational modeling for business processes engineering and workflows [5]. But practically UML activity diagrams can be used to model business processes, or the dynamic part of a model (e.g. an algorithm of an operation). They can represent a process, or the behavior of an operation.
A comparison between the Business Process Diagram (BPD) and the UML Activity Diagrams for modeling of twenty-one workflows patterns are introduced in [6]. It shows enough similarity between the two diagrams in terms of notations and the representations. However, other benefits are provided by the BPMN, its mathematical foundations designed to easily transform it in a business language. Furthermore, BPMN can be translated into UML and provide a solid modeling mean.

\subsection{Related Work}

Several methods of modeling and transformation of models have been proposed in the context of the MDA. However, through the bibliographical study that we have conducted only seven methods seem to address the modeling of the CIM level and its transformation to the PIM level.

The authors in [7] proposed a disciplined method for transformation of CIM to PIM. The CIM model uses two activity diagrams to represent business processes and system requirements. The business process model represents all the activities of the organization independently of their automation; while the requirements model specifies the system supporting such activities, by representing their use cases and considering it as a one actor. The PIM model is represented by the class diagram obtained from the requirements model. This last model is transformed into component models of the system that provide a first sketch of the structure of the system: a set of business archetypes that helps to transform, in detail, the components system into PIM. This approach is based on modeling the CIM using the UML 2.0 Activity Diagrams as a single technique, and the PIM behavioral aspect is not specified.

The method in [8] proposed a CIM to PIM transformation using a method oriented by the features and based on components. The requirements in the CIM model are represented by a model that includes a set of features and relations between them. And the PIM model is represented by a software architecture that includes a set of components and their interactions. This method uses an intermediate model that is neither CIM nor PIM, which does not consider business processes.

In the papers $[9,10,11]$, the authors present a CIM composed by a business processes model, using a secured business process with the BPMN. This CIM is transformed, with the help of the QVT rules (Query/View/Transformation), checklists, and refinement rules into two models composing the PIM level: the use cases diagram and the class diagram. The use cases are detailed in order to obtain the activities and the class diagram that is considered an initial analysis model. Use cases diagram is moved, in this method, into the PIM level. Furthermore, the diagrams of the PIM that are obtained by transformation of the CIM, do not communicate the PIM behavioral structure.

Paper [12] presents an analytical solution for the 
modeling of CIM and its transformation to the PIM model. To model business processes, the authors used at the CIM level the Data Flow Diagrams (DFD). Whereas the PIM level is covered by four UML diagrams: The Use Cases Diagram, The Activities Diagram, The Sequence Diagrams and the Domain Models.

Paper [13] tackles a semi-automatic method for building web applications from the high level of requirements, expressed as use cases in accordance with the model-driven architecture (MDA). The first step of the method is to transform the CIM model to the PIM model. It considers that the CIM is represented by the description of use cases as well as the default domain objects. The PIM model includes State Machines, the User Interface Model and the refined domain model. This method does not consider business processes.

In paper [14] the authors present a systematic method for MDA transformations, including the creation of the platform independent model (PIM) from the CIM, the transformation of the PIM to the platform specific model (PSM) and the generation of code from the PSM model. The CIM in this method is composed of the use cases diagram, the activity diagram and the robustness diagram. While, the PIM is modeled by two parts: the behavioral part by a sequence diagrams and the structural part by a class diagram.

The method in [15] allows, first, to build the CIM model and to transform it (semi-) automatically to the less abstraction level (PIM).The CIM level is covered by two models; on one hand the business process model (BPM) characterizing both the behavioral and the static aspects representing the different activities and resources used by them in the business processes. On the other hand, the use cases model representing functional aspect of system. While, the PIM level is modeled with the domain classes diagram (DCD) and the external behavior sequence diagram of the system (SDSEB). The later is an UML sequence diagram that demonstrates interactions between the actors and the system seen as unique entity represented by a one line of life, without focus on the interactions of the objects system. This method calls the business rules to generate the DCD PIM level.

It should be noted that in the literature we have found two other proposals that are limited only to the modeling of the CIM level, without giving details on its transformation into PIM. The method in paper [16] presents a method for modeling CIM based on the artifacts and the concepts of the RUP methodology. This method presents a CIM which covers two aspects: the business processes and requirements. It is composed of three models: A business use cases model, a business analyses model and the use cases model. Moreover, the method in the papers [17, 18, 19], called TFMfMDA (Topological Functioning Modeling for Model Driven Architecture) using formal mathematical foundations of topological functioning model. The CIM level is modeled with use cases model and the conceptual class diagram presenting the domain concepts and their relations to establish.

\section{CIM Architecture}

The creation of the CIM is the first task in the MDA development process. It must be developed in collaboration with the domain experts. The CIM is of great importance for the rest of the development process; any changes driven by new requirements at the CIM level will reflect the PIM and the PSM levels.

\subsection{Specifications of CIM According to the OMG}

According to the definition given by the OMG [1], we have deduced that the CIM model must verify the following requirements:

- CIM represents a point of view of the system independently of the computation.

- CIM should not show the details of the structure of the system.

- CIM is sometimes called domain model or a business model, using for its specification, the current vocabulary of practitioners of domain in question.

- Since the primary user of the CIM -the practitioner of domain- is generally not a know-all of models or of the artifacts used to make features for which the requirements are identified in the CIM. The CIM must play an important role in bridging the gap between the domain experts with its requirements and the experts of the design and construction of objects which must satisfy the domain requirements.

- The system requirements are modeled in the CIM.

- CIM describes the situation in which the system will be used.

- CIM is independent of the system implementation. It hides many or all of the information on the use of data automatic processing systems.

- CIM represents a source of common vocabulary to use in other models of the MDA.

- The requirements of the CIM must be traceable to the artifacts of the PIM and the PSM, and vice versa.

- CIM can include several models, some providing more details than others or focused on specific preoccupations.

\subsection{Architecture of CIM in our Approach}

According to Xavier Blanc [20], in a wider context, the requirements model (CIM) is considered as a complex entity, constituted by a glossary, the definitions of business processes, the requirements, and the use cases as well as a systemic view of application.

By analyzing the related works previously presented, there is no consensus on the number and types of elements constituting the CIM. In order to propose an appropriate CIM from previous requirements, our proposal suggests that the CIM must compose of two models: the BPMN Diagram, to describe the different business processes represented by different sequences of activities, and the use cases diagram 
(UCD) allowing in turn the description of the requirements in the CIM level.

The system, at this level, is seen as black box to ensure the independence of any computation by hiding the details of the structure of the system.

A refinement between the two models (BPMN and UC) allows us, on one hand, to have the same vision between domain experts and technological ones and on the other hand to validate the CIM level before any transformation into the low levels of MDA.

\subsubsection{Business Process Diagram (BPD)}

A business process represents the interactions in the form of exchange of information between various actors: humans, applications or services and third-party processes.
Business Process Model and Notation (BPMN) is a graphical notation used to specify and to model business processes. Its main objective is to provide a standard notation that is easily understandable by all actors of the organization. It is a business model using a simple notation for domain practitioners, facilitating communication between experts of the domain and requirements, and technical experts. It shows the system in its environment.

The BPMN model called BDP (Business Process Diagram) consists of a small set of graphic elements classed into four categories: Flow Objects, Connecting Objects, Swimlanes and Artifacts [21].

Figure 3 illustrates the main fragment of the BPMN metamodel.



Figure 3. Main fragment of the meta-model BPMN

\subsubsection{Use Cases Model (UML UC)}

Use case diagrams allow identifying the features of a system and the conditions for their good functioning. They show functional elements, actors and objects in interaction. To this end, a use case diagram contains actors and use cases.

An actor is an entity that can interact with the system; whereas, a use case is a set of interactions between some actors and the system under development.

UML (Unified Modeling Language) defines a use case as : "the specification of a set of actions performed by a system, which yields an observable result that is, typically, of value for one or more actors or other stakeholders of the system" [5].

The use cases are not enchained. There is no temporal representation in a use case diagram. Each use case can be described in detail, describing the interactions between actors and the system, and the order in which they occur.
Several techniques exist for the detailed description of a use case, such as state machines, activity diagrams, or informal text. For our proposed method, we use the textual description (TD) of use cases that we formalize using SBVR (Semantic Business Vocabulary and Business Rules) [22].

We adopt the use cases diagrams for several reasons:

- Use cases diagrams play a very important role for the identification of the requirements of the users. They describe exhaustively the functional requirements of the system.

- Because of their simplicity, the analysts and the developers are familiar with the use of the use cases diagrams.

- The existence of the development process guided by the different cases, such as the Unified Process (UP) described by the authors of UML [23] and the Rational Unified Process (RUP) [24] that adheres to good 
development practices observed in the industry for their success. diagram is shown in figure 4, and the figure 5 illustrates the main fragment of the SBVR metamodel.

A simplified version of the metamodel of a use case



Figure 4. Main fragment of the meta-model UC

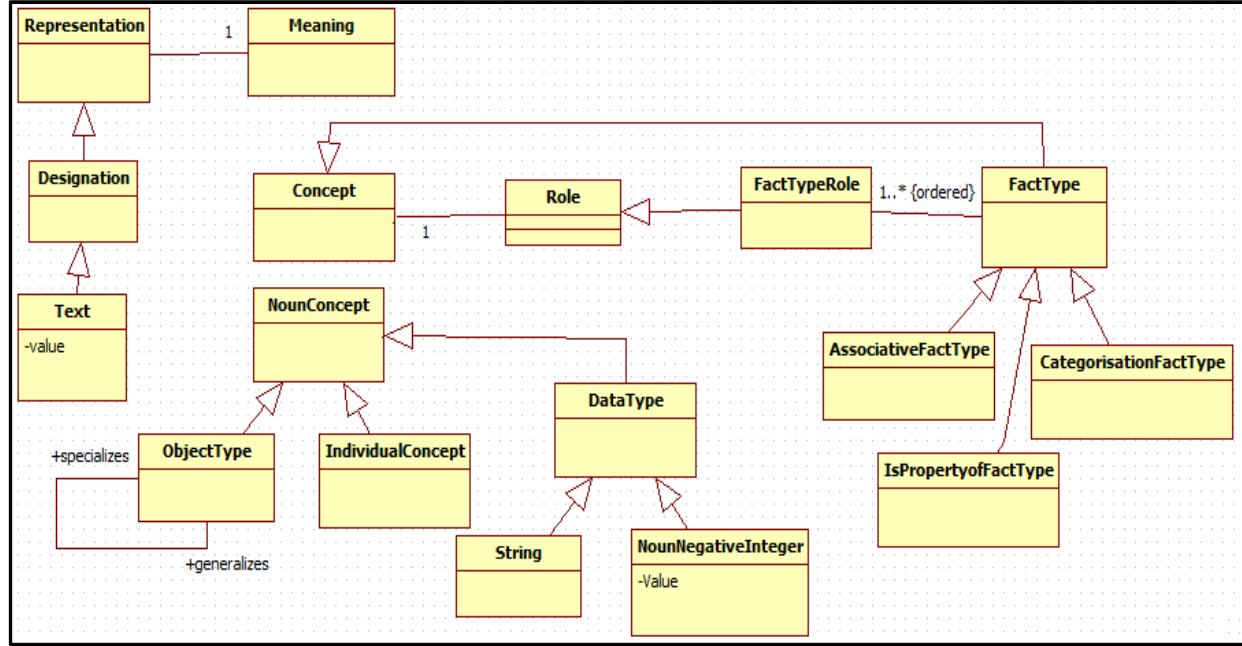

Figure 5. Main fragment of the meta-model SBVR

\section{PIM Architecture}

In previous section, we have presented the structure of the CIM proposed for our method, but the goal of our approach does not stop here. It must identify also the structure of the PIM, which can be used as a result of the development process, and define the transformation enabling to generate it automatically from the CIM. In this section, we define the adequate structure of the PIM that can easily integrate the development process. This PIM should be generated automatically through a transformation mechanism that we are going to define in section 5. Also, it must be generic capable of being transmitted to the PSM model.

\subsection{Specifications of the PIM Model According to the OMG}

According to the OMG [1], the PIM model must meet the following requirements:

- The PIM represents a viewpoint of the system independently of any platform. Therefore may be appropriate for use with the similar platforms.

- The PIM describes the system, but doesn't show details on the use of its platform.

- A PIM can be adapted to a particular architectural style or more.

- The independence of PIM with respect platform can be achieved using a "technology-neutral virtual machine".

- The PIM will be transformed to the PSM.

In summary, the PIM is a view of a system without any knowledge of the implementation details. It describes the information system, but hides the details on the use of technologies that will be used to deploy the application. There are several levels of PIM but all are independent of any platform. PIM can integrate technological and architectural aspects but always without platform-specific 
details. It can contain, for example, information about security, persistence, etc. That allows more precisely to project the PIM model towards a specific model PSM.

\subsection{Architecture of the PIM Model in Our Approach}

The PIM, called Model of analysis and design, represents the business logic specific for a system. It depicts the functioning of entities and services. It must be sustainable over time.

The UML language is imposed today as a reference for realizing all analysis and design models. At this level we are interested in an abstract design, realizable without any knowledge of the techniques implementation. Thus, the application of the design patterns or the GoF (Gang of Four) is part of this stage of design. Nevertheless, the application of technical patterns, specific for some platforms, corresponds to a next step [20].

So, an adequate PIM should represent two aspects of the system:

- The structural aspect (static) of the system using classes, objects, attributes, operations, relations, etc.

- The behavioral aspect of the system showing the interactions between objects, etc.

At this level, the formalism used to express the PIM is a domain class diagram coupled with a system sequence diagram expressed in UML.

\subsubsection{Domain Classes Diagram (DCD)}

A domain model is not a description of software objects but a visualization of the concepts of a real-world domain. We speak about the analysis objects. It is possible that an analysis object becomes a software object during the design, but this is not systematic.

Domain class diagram should not be confused with a design class diagram. A domain class diagram can be enriched with methods obtained from the different interaction diagrams, such as the sequence diagram or a state-machine diagram for obtaining the design class diagram that contains classes with the signatures of their methods.

An UML class diagram will be used to represent the domain model. It can contain only the classes and some attributes without specification of the operations.

The domain class diagram represents in our approach the static view of the PIM.



Figure 6. Simplified metamodel of Domain Class Diagram

\subsubsection{System Sequence Diagram (SSD)}

The objective of the system sequence diagrams (DSS) is to describe the behavior of the system where it is seen as a 'black box' (from the analysis point of view). The system is thus seen from the outside (by actors) without prejudice to how it will be achieved. The 'black box' will be open (described) to a subsequent design phase. The process of a use case will be described as a sequence of messages exchanged between the actors and the system.



Figure 7. Main fragment of the System Sequence Diagram Meta-model



Figure 8. Overview of the process of transformation of the CIM to PIM

\section{Steps of Transformation Approach from CIM to PIM}

This section presents the approach for CIM modeling and its transformation to the PIM model.

Our approach consists of three steps:

- Step 1: Modeling CIM through the BPMN model (BPD) and the of use cases model (UML UC) obtained by the horizontal transformation CIM2CIM (figure 9) from the first model BPD.

- Step 2: Obtain from the CIM, the behavioral view of the PIM represented by a System Sequence Diagram (UML SSD). This step is assured by the vertical transformation CIM2PIM noted C2P1 in the remainder of the paper, as shown in figure 8 . This step has been detailed in our 
contribution [25].

- Step 3: Generate the static view of the PIM that is represented by a Domain Class Diagram (UML DCD) from the CIM. This part of the PIM is the result of a vertical transformation CIM2PIM designated C2P2 in this paper, as shown in figure 8 . This step was presented in our article [26].

Any stage of transformation in our approach will be carried out as follows:

- Defining the rules of transformations of the source to the model target.

- Expression of the transformation rules.

- Application of transformation rules.

Thus, the transformation process takes as input a source model, performs the transformation rules and produces output as a target model.

It has opted for the use of the QVT standard for the expression of the transformation rules. An example of written language QVT transformation rule is presented as an example in table 99.

\subsection{CIM Modelling}

At the CIM level, two essential roles are distinguished. The business analyst is responsible for developing business processes diagrams without taking into account the technical aspects, and the computer expert that takes the responsibility to add the information necessary for the transcription of models in an execution language.

The CIM modeling begins with the representation of business processes via a BPMN diagram, then its horizontal transformation towards a use cases diagram detailed (UML $\mathrm{UC})$, through the $\mathrm{C} 2 \mathrm{C} 1$ transformation.

In order to have a common understanding of the system by business experts and technical experts, and do not drop system requirements, we propose a CIM refinement by a second exogenous horizontal transformation $\mathrm{C} 2 \mathrm{C} 2$ of the UML-UC model to BMPN model (BPD). By this step, we try to validate the CIM model before any vertical transformation to the PIM level.

\subsubsection{Business Processes Representation in Our Approach: BPD}

For our approach, business processes are supposed to be defined. They should be formalized (modeled) if they are not already realized. We propose for that purpose to use the BPMN specification for modeling the business processes. It is done by grouping the components of the various business processes in one diagram of process BPD (Business Process Diagram).

\subsubsection{Transformation from BPD to UCD: $C 2 C 1$}

The $\mathrm{C} 2 \mathrm{C} 1$ is a horizontal and exogenous transformation that consists of transforming a BPMN model conforms to its metamodel towards a use cases model conforms to its metamodel.

This transformation is based on transformation rules written in the QVT standard. The transformation process takes as input a source model, executes the transformation rules and produces output as a target model. This consists of:

- Defining a mapping between the elements of the BPD and the elements of the use cases diagram.

- Performing the mapping using the model transformation language QVT.

The proposed mapping rules are based on the equivalence between the BPMN concepts and the UC concepts. The mapping used for this transformation is presented in table 1.

A detailed description of the first rule presented in table 2 .

Table 1. BPMN2UC Transformation Rules

\begin{tabular}{|c|c|c|c|}
\hline Rule & Transformation Rule & Source Model Element & Target Model Element \\
\hline 1 & Pool2Actor & Pool & Actor \\
\hline 2 & Lane2Actor & Lane & Actor \\
\hline 3 & Lane. within. Pool 2 Generalization & Pool that contain Lanes & Actor(Pool) is a generalization of Actor(Lane) \\
\hline 4 & Activity2UC & Activity (Sub-Process or Activity of type task) & Use Case \\
\hline 5 & $\begin{array}{l}\text { Activity. In. Swimlane } \\
\text { 2Association }\end{array}$ & $\begin{array}{l}\text { Activity (Sub-Process or Activity of type task) } \\
\text { within a swimlane }\end{array}$ & $\begin{array}{l}\text { Association between actor corresponding to swimlane } \\
\text { and UC corresponding to Activity }\end{array}$ \\
\hline 6 & $\begin{array}{l}\text { Sequence Flow Or Message Flow } 2 \\
\text { include }\end{array}$ & Flow & include \\
\hline 7 & Gateway2extend & Decision Gateway & Extend between the related activities \\
\hline \multicolumn{4}{|c|}{ For each activity type sub-processes } \\
\hline 8 & $\begin{array}{l}\text { Task. In. Actor. Swimlane } \\
2 \text { Message From Actor ToSys }\end{array}$ & Task in Actor swimlane performed by Actor & System Event (Message sent to System) \\
\hline 9 & $\begin{array}{l}\text { Task. In. System. Swimlane } 2 \text { System } \\
\text { Response }\end{array}$ & Task in System Swimlane oriented Actor & $\begin{array}{l}\text { System Response (Response Message from the } \\
\text { System to Actor) }\end{array}$ \\
\hline \multicolumn{4}{|c|}{ After each Decision Gateway within sub-processes } \\
\hline 10 & Successful Flow 2 Event & Successful Flow & System Event Or System Response (Rules 8 \& 9) \\
\hline 11 & $\begin{array}{l}\text { Alternative Flow } \\
2 \text { Alternative Scenario }\end{array}$ & $\begin{array}{l}\text { Alternative Flow that terminate Sup-Process } \\
\text { correctly }\end{array}$ & Alternative Scenario \\
\hline 12 & $\begin{array}{l}\text { Error Flow } \\
2 \text { Error Scenario }\end{array}$ & $\begin{array}{l}\text { Error Flow that terminate Sup-Process with } \\
\text { errors }\end{array}$ & Error Scenario \\
\hline
\end{tabular}


Table 2. QVT code of the first rule



\subsubsection{Transformation from UCD to BPD: C2C2}

The $\mathrm{C}_{2} \mathrm{C}_{2}$ is a horizontal and exogenous transformation that consists of transforming a use cases model conforms to its meta-model to a BPMN model conforms to its metamodel. Table 3 summarizes the rules used for $\mathrm{C}_{2} \mathrm{C}_{2}$ transformation.

Table 3. Use-Case to BPMN transformation QVT rule

\begin{tabular}{llll}
\hline Rule & Transformation Rule & Source Model Element & Target Model Element \\
\hline 1 & Actor2Lane & Actor & Lane \\
2 & UC2Activity & Use Case & Activity (Sub-Process or Activity of type task) \\
3 & Include2 SequenceFlow & include & Sequence Flow \\
4 & Include 2MessageFlow & Include & Message Flow \\
\hline
\end{tabular}

\subsection{Obtaining the PIM Behavioral Model from the CIM}

The use case model obtained in the first step constitutes the source of the CIM2PIM transformation, subsequently noted $\mathrm{C}_{2} \mathrm{P}_{1}$, allowing the production of the model representing the dynamic part of the PIM.

$\mathrm{C}_{2} \mathrm{P}_{1}$ is an exogenous and vertical transformation which consists of transforming the UML-UC that is conformed to its metamodel to a system sequence model which is conformed to its metamodel. Thus, this transformation consists of defining a mapping between the elements of the UC model and elements representing the system sequence diagram, and then to express the corresponding QVT transformation rules. The proposed mapping rules are based on the equivalence between the concepts of UML UC and the concepts of the SSD. This mapping is presented in table 4 .

Table 4. UC2SSD Transformation Rules

\begin{tabular}{llll}
\hline Rule & Transformation Rule & Source Model Element & Target Model Element \\
\hline 1 & UseCase2SSD & Use Case & SSD \\
2 & Principal Actor 2Actor & Actor that directly operates on the System & Actor \\
3 & System Event 2 System Message & System Event (Message sent to System) & Message sent From Actor to System \\
4 & Sys Response & System Response (Response Message from & Message sent From System to Actor \\
5 & 2SystemMessage & the System to Actor) & Internal System Message \\
6 & Internal Task 2 Internal Message & Non-related system task to actor & Interaction Fragment 'Alt' \\
7 & Alternative Scenario 2 Alt & Alternative Scenario & Interaction Fragment 'Break' \\
\hline
\end{tabular}

\subsection{Obtaining the PIM Static Model from the CIM}

The CIM2PIM transformation, noted subsequently $\mathrm{C}_{2} \mathrm{P}_{2}$, is defined by 6 transformation rules illustrated in table 4 . This exogenous and vertical transformation aims at transforming the BPMN model that is conformed to its metamodel to a domain classes model (UML DCD) which is conformed to its metamodel. It specifies how one or more elements of the BPMN model (source model) are 
transformed into one or more elements of the UML-DCD model (target model).

The proposed mapping rules are based on the equivalence between the concepts of the BPMN and the concepts of the DCD. This mapping is presented in table5.

Table 5. BPMN2DCD Transformation Rules

\begin{tabular}{|c|c|c|c|}
\hline Rule & Transformation Rule & Source Model Element & Target Model Element \\
\hline 1 & Pool 2 Class & Pool & Class \\
\hline 2 & Lane 2 Class & Lane & Class \\
\hline 3 & Lane. within. Pool 2 Aggregation & Pool that contain Lanes & $\begin{array}{l}\text { Aggregation relationships between the Class derived from Pool } \\
\text { And the Classes derived from Lanes }\end{array}$ \\
\hline 4 & DataObject2Class & Data Object & Class \\
\hline 5 & Activity 2 Operation & Activity & $\begin{array}{l}\text { Operation attached to the corresponding analysis class at the } \\
\text { container (Lane, Pool or Group) }\end{array}$ \\
\hline 6 & Group 2 Class & Group & Class \\
\hline
\end{tabular}

\section{Case Study}

With the aim to illustrate our method by an example, we have taken our case study presented in our previous works $[25,26]$. This example is based on a business process of enrollment system for training in a school. It models the interaction between customers and the school.

We have a one swimlane corresponding to 'customers' and another one for the 'school side'. However, since there are two actors involved in the former, we use a pool with two lanes, one for each actor. Thus, within the school pool there is a lane for an 'Assistant' and another for 'Financial Services'. Figure 9 depicts the process of our case study, whereas figure 10 presents the detail of the first Sub-Process 'Choose Training' using Data Objects as an example. It employs two Pools, one for a customer and another one for a system. In the same way we can represent the other subprocesses: 'Order Training', 'Payment', and 'Training Schedule',



Figure 9. Business Process Diagram of the case study "enrollment system for training"



Figure 10. Diagram of Sub-Process “Choose Training” 
Table 6. BPMN2UC Transformation Rules for the case study

\begin{tabular}{|c|c|c|}
\hline Rule & & Use Cases Element \\
\hline 1 & Pool 2 Actor & Customer, School Area \\
\hline 2 & Lane 2 Actor & Assistant, Financial Services \\
\hline 3 & $\begin{array}{l}\text { Lane. within. Pool } \\
2 \text { Generalization }\end{array}$ & 'School Area' Actor is a generalization for 'Assistant' actor and 'Financial Services' actor \\
\hline 4 & Activity 2 UC & $\begin{array}{l}\text { Choose Training, Order Training, Pay Training, Receive Training Schedule, Receive Choice, } \\
\text { Receive Order Training, Send Schedule, Deliver Quotation, Validate Payment }\end{array}$ \\
\hline 5 & $\begin{array}{l}\text { Activity. In. Swimlane } \\
2 \text { Association }\end{array}$ & $\begin{array}{l}\text { Each UC is associated at the Actor corresponding to the Swimlane. (For example : 'Choose } \\
\text { Training' is associated at 'Customer') }\end{array}$ \\
\hline 6 & Sequence Flow Or Message Flow 2 include & $\begin{array}{l}\text { 'Receive Choice' include 'Choose Training' } \\
\text { 'Deliver Quotation' include 'Receive Choice", ... } \\
\text { 'Choose Training' extend 'Order Training' }\end{array}$ \\
\hline 7 & Gateway 2 extend & $\begin{array}{l}\text { 'Order Training' extend 'Pay Training' } \\
\text { 'Validate Payment' extend 'Send Schedule' }\end{array}$ \\
\hline 8 & $\begin{array}{l}\text { Task. In. Actor. Swimlane } \\
2 \text { Message From Actor ToSys }\end{array}$ & $\begin{array}{l}\text { Request Training Catalog } \\
\text { Fill Form Training needs } \\
\text { Quotation Request }\end{array}$ \\
\hline 9 & $\begin{array}{l}\text { Task. In. System. Swimlane } 2 \text { System } \\
\text { Response }\end{array}$ & Deliver Training Catalog, Accepted Form Training, Quotation Deliver \\
\hline 10 & Successful Flow 2 Event & Choice in Catalog \\
\hline 11 & $\begin{array}{l}\text { Alternative Flow } \\
2 \text { Alternative Scenario }\end{array}$ & Specific Choice \\
\hline 12 & Error Flow2 Error Scenario & Cancel Choice \\
\hline
\end{tabular}

The Use Cases Diagram obtained by applying the $\mathrm{C}_{2} \mathrm{C}_{1}$ transformation rules is shown in figure 11 below. Table 12 shows the $\mathrm{C}_{2} \mathrm{P}_{1}$ transformation rules corresponding to a Sub-Process "choose training".



Figure 11. Use Cases Diagram of the Case Study 
Table 7. UC2SSD Transformation Rules for the case study corresponding at the UC "Choose Training"

\begin{tabular}{rll}
\hline Rule & & SSD Element \\
\hline 1 & Use Case 2 SSD & SSD_Choose_Training \\
2 & Principal Actor 2 Actor & Customer \\
& System Event & se1. Request Training Catalog() \\
3 & 2System Message & se2. Form Training Needs() \\
& & se3. Quotation Request() \\
& Sys Response & sr1. Deliver Training Catalog() \\
4 & 2 System Message & sr2. Accepted Form Training() \\
& Internal Task 2 Internal Message & sr3. Qoutation Deliver() \\
6 & Alternative Scenario 2 Alt & se2.1. Analyze Form() \\
7 & Error Scenario 2 Break & Interaction Fragment 'Alt' \\
\hline
\end{tabular}

Figure 13 depicts the SSD obtained from a Use Cases Diagram by applying the C2P1 transformation rules.



Figure 12. The SSD of the Pay Training Use Case

The C2P transformation rules corresponding to our case study are presented in table 8 below.

Table 8. BPMN2DCD Transformation Rules for the case study

\begin{tabular}{cll}
\hline Rule & & DCD Element \\
\hline 1 & Pool 2 Class & Customer \\
2 & Lane 2 Class & Assistant, Financial Service \\
3 & Lane. within. Pool 2 & Aggregation relationships between School Area And Assistant \\
& Aggregation & Aggregation relationships between School Area And Financial Service \\
& & Request Training Catalog, \\
& & Training Catalog, \\
& & Form Training Needs, \\
& & Quotation Request, \\
& Data Object 2 Class & Quotation, \\
& & Order Training, \\
& & Payment, \\
& & Training Schedule \\
& & Operations of Customer : \\
& & Choose Training(), Order Training(), \\
& & Receive Training(), \\
& & Operations of Assistant : \\
& & Receive Choice(), Receive Order Training(), \\
& Activity 2 Operation & Send Training(); \\
& & Operations of Finance Service : \\
& & Deliver Quotation(); Validate Pay() \\
\hline
\end{tabular}


Figure 14 show the Domain Class Diagram obtained by applying the C2P2 transformation rules.

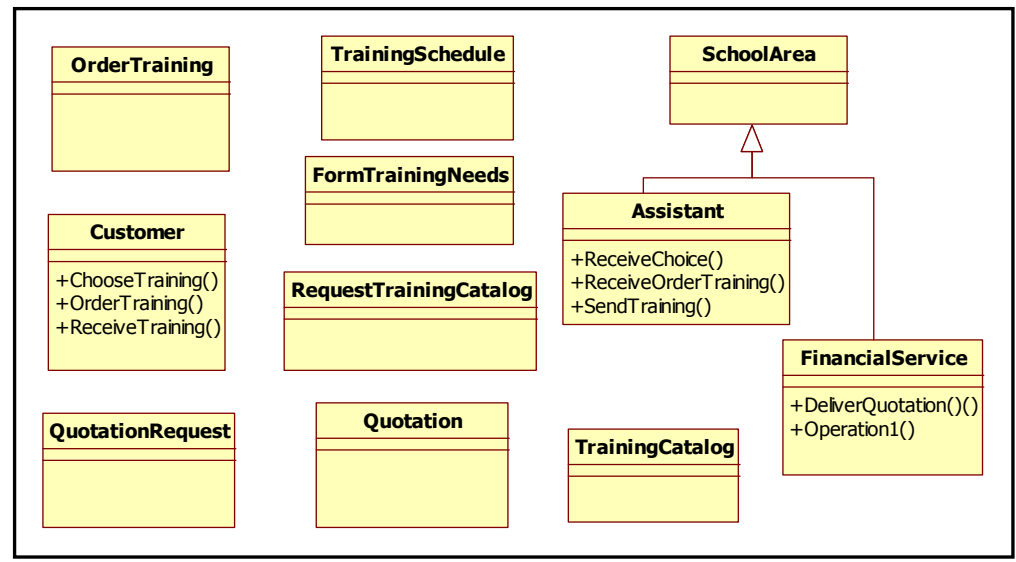

Figure 13. DCD of Sub-Process "Choose Training"

\section{Analysis and Evaluation}

According to our previous work [27], we have announced that an ideal method for modeling the CIM and its transformation into PIM should have the following characteristics:

- The CIM modeling should cover the different views of the business domain: static, dynamic and functional.

- Generating the PIM model -representing the structural and behavioral aspects of the system- from the CIM
- The CIM to PIM transformation should be automated, taking into account the traceability and offering the entire transformation rules.

The evaluation is based on the criteria that we have defined in the paper [27] in order to evaluate different methods which propose to model CIM and transform it into PIM.

The results of the evaluation are presented in table 9. The rows in the table design the studied methods and each column in the table represents an evaluation criterion.

Table 9. Results of the evaluation

\begin{tabular}{|c|c|c|c|c|c|c|c|c|c|}
\hline \multirow[t]{2}{*}{ Methods Studied } & \multicolumn{3}{|c|}{ CIM coverage } & \multicolumn{2}{|c|}{ PIM completeness } & \multicolumn{4}{|c|}{ CIM to PIM transformation } \\
\hline &  &  & 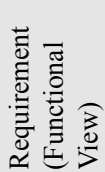 & 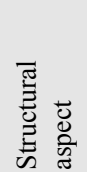 &  &  &  &  &  \\
\hline Kherraf and al. [7] & $\mathrm{N}$ & $\mathrm{Y}$ & $\mathrm{Y}$ & $\mathrm{Y}$ & $\mathrm{N}$ & $\mathrm{P}$ & $\mathrm{P}$ & 1 & a \\
\hline Bousetta and al. [15] & $P$ & Y & $\mathrm{Y}$ & Y & $\mathrm{Y}$ & $\mathrm{P}$ & $\mathrm{P}$ & $\mathrm{I}$ & ? \\
\hline Kardoš and al. [12] & $\mathrm{N}$ & $\mathrm{P}$ & $\mathrm{N}$ & Y & $\mathrm{Y}$ & $\mathrm{P}$ & $\mathrm{N}$ & r & N \\
\hline Rodríguez and al. $[9,10,11]$ & $\mathrm{N}$ & Y & $\mathrm{Y}$ & Y & Y & $\mathrm{P}$ & $\mathrm{P}$ & $\mathrm{I}$ & ? \\
\hline Wu and al.[14] & $\mathrm{P}$ & Y & $\mathrm{Y}$ & Y & Y & $\mathrm{N}$ & $\mathrm{N}$ & $\mathrm{I}$ &  \\
\hline Zhang and al. [8] & $\mathrm{N}$ & $\mathrm{Y}$ & $\mathrm{N}$ & Y & $\mathrm{N}$ & $\mathrm{P}$ & $\mathrm{Y}$ & I & ? \\
\hline Fatolahi and al. [13] & $\mathrm{Y}$ & $\mathrm{N}$ & $\mathrm{Y}$ & Y & Y & $\mathrm{P}$ & $\mathrm{N}$ & 1 & v \\
\hline Sharifi and al. [16] & $\mathrm{Y}$ & $\mathrm{N}$ & $\mathrm{Y}$ & & & & & & \\
\hline Erika and al. $[17,18,19]$ & $\mathrm{Y}$ & $\mathrm{N}$ & $\mathrm{Y}$ & & & & & & \\
\hline
\end{tabular}

Legend: Y: Yes; N: No; P: Partial

By analyzing the different results obtained, we conclude that only the method of the paper [15] which remains close to the ideal description of a method allowing the building of the CIM and transforming it to the PIM. Our proposal complements this method, by satisfying all the requirements needed during the construction of the CIM and its transformation into PIM.

\section{Conclusion and Future Work}

This paper proposes an approach for CIM modeling and its transformation into PIM based on three steps:

- Representing the CIM by BPMN model and use cases model that covers the static, the behavioral and the functional views of the system. The transition from one model to another is provided by a horizontal model transformation.

- Obtaining from the CIM the behavioral view of the PIM. This step is assured by a vertical transformation from use cases model to a system sequence diagram.

- Generating from the PIM the static view of the PIM. This step is the result of a vertical transformation from 
BPMN model to a Domain Class Diagram.

In order to make transformations automatic, we have developed all the transformation rules involved in this paper using the QVT language.

Future works aim at developing a tool that supports all the transformations performed in this paper.

\section{References}

[1] J. Miller and J. Mukerji, "MDA Guide Version 1.0.1.," OMG, 2003.

[2] R. Soley, «Model driven architecture (mda), draft 3.2. Rapport technique, disponible sur: http://www.omg.org/cgibin/doc?omg/00-11-05,» 2000.

[3] OMG, "Meta Object Facility (MOF)2.0 Query/View/Transformation Specification, http://www.omg.org/spec/QVT/1.0/PDF," 2009.

[4] S. A. White, "Introduction to BPMN," IBM Cooperation, pp. 2008-029, 2004.

[5] OMG, "OMG Unified Modeling LanguageTM (OMG UML), Superstructure,

http://www.omg.org/spec/UML/2.4.1/Superstructure," August 2011.

[6] S. A. White, "Process Modelling Notations and Workflow Patterns, http://www.omg.org /bp-corner/bpfiles/Process_Modeling_Notations.pdf," IBM Corporation, 2004.

[7] S. Kherraf, E. Lefebvre and W. Suryn, "Transformation from CIM to PIM Using Patterns and Archetypes," in 19th Australian Conference on Software Engineering, 2008.

[8] W. Zhang, H. Mei, H. Zhao and J. and Yang, "Transformation from CIM to PIM: A Feature-Oriented Component-Based Approach," in Model Driven Engineering Languages and Systems volume 3713 of Lecture Notes in Computer Science, pages 248-263, Springer Berlin / Heidelberg, 2005.

[9] A. Rodríguez, E. Fernández-Medina and M. Piattini, "Towards obtaining analysis-level class and use case diagrams from business process models," Advances in Conceptual ModelingChallenges and Opportunities. Springer Berlin Heidelberg., pp. 103-112, 2008.

[10] A. Rodríguez, I. G.-R. de Guzmán, E. Fernández-Medina and M. Piattini, "Semi-formal transformation of secure business processes into analysis class and use case models: An mda approach," in Information and Software Technology, 52(9):945- 971, 2010.

[11] A. Rodríguez, E. Fernández-Medina, J. Trujillo and M. Piattini, "Secure business process model specification through a UML 2.0 activity diagram profile," Decision Support Systems, vol. 51, no. 3, pp. 446-465, 2011.

[12] M. Kardoš and M. Drozdová, "Analytical method of CIM to PIM transformation in Model Driven Architecture (MDA)," Journal Of Information And Organizational Sciences, vol. 34, pp. 89-99, 2010.
[13] A. Fatolahi, S. S. Somé and T. C. Lethbridge, "Towards a semi-automated model-driven method for the generation of web-based applications from use cases," in 4th Model Driven Web Engineering Workshop (p. 31), 2008.

[14] J. H. Wu, S. S. Shin, J. L. Chien, W. S. Chao and M. C. Hsieh, "An extended MDA method for user interface modeling and transformation," in The 15th European Conference on Information Systems (pp. 1632-1641)., June 2007.

[15] B. Bousetta, O. El Beggar and T. Gadi, "A methodology for CIM modelling and its transformation to PIM," Journal of Information Engineering and Applications, vol. 3, no. 2, pp. 1$21,2013$.

[16] H. R. Sharifi and M. Mohsenzadeh, "A New Method for Generating CIM Using Business and Requirement Models.," World of Computer Science and Information Technology Journal (WCSIT), vol. 2, no. 1, pp. 8-12, 2012.

[17] J. Osis, E. Asnina and A. Grave, "Computation Independent Modeling within the MDA," in Software-Science, Technology \& Engineering, 2007. SwSTE 2007. IEEE International Conference on (pp. 22-34). IEEE, October 2007.

[18] J. Osis, E. Asnina and A. Grave, "Formal computation independent model of the problem domain within the MDA," in Proceedings of the 10th International Conference on Information System Implementation and Modeling (pp. 23-25), April 2007.

[19] J. Osis, E. Asnina and A. Grave, "Computation independent representation of the problem domain," in MDA. J. Software Eng, 2(1), 19-46., 2008.

[20] B. Xavier, MDA en Action : Ingénirie Logicielles Dirigée par les Modèles, Paris: Eyrolles, 2005.

[21] OMG, «Business Process Model and Notation (BPMN), Version 2.0.1,» OMG, http://www.omg.org/spec/BPMN, September 2013.

[22] OMG, "OMG Unified Modeling LanguageTM (OMG UML), Superstructure,

http://www.omg.org/spec/UML/2.4.1/Superstructure," August 2011.

[23] OMG, «Semantics of Business vocabulary and Rules,» 2008. [En ligne].

[24] I. Jacobson, G. Booc et J. Rumbaugh, Le Processus unifié de développement logicie, Eyrolles, 2000.

[25] P. Kruchte, The rational Unified Process An introduction, Addison-WESLEY, 2000.

[26] A. Kriouile, T. Gadi, N. ADDAMSSIRI et A. El Khadimi, «Obtaining behavioral model of PIM from the CIM,» Multimedia Computing and Systems (ICMCS), 2014 International Conference on. IEEE, pp. 949-954., 2014.

[27] A. Kriouile, N. Addamssiri, T. Gadi et Y. Balouki, «Getting the Static Model of PIM from the CIM,» chez 3rd Colloquium IEEE on Information Science and Technology (CiSt'14), Tetuan, 2014.

[28] A. Kriouile, T. Gadi and Y. Balouki, "CIM to PIM Transformation: A criteria Based Evaluation," IJCTA, vol. Vol 4 (4), no. ISSSN:2229-6093, pp. 616-625, July-August 2013. 